\title{
Disaster Medicine: Fact or Fiction
}

\author{
Marvin L. Birnbaum, MD, PhD
}

As I ponder Disaster Medicine, several questions repeatedly surface: What is it? Who should do it? Is it special? How does Disaster Medicine differ from other areas of medicine? What are our reasons to be; our missions?

Most of these questions are addressed in the Guest Editorial by Manni and Magalini. They point out that the practice of Disaster Medicine demands a special set of skills and knowledge. Special medical abilities, including preventive and public health, must be coupled with a host of non-medical capabilities. Thus, those of us who have a keen interest in Disaster Medicine must develop a knowledge base and skills which are outside the current realm of medicine.

Unfortunately, it is difficult to acquire the experience necessary to develop expertise for the practice of this element of medical practice. For most physicians, the setting of a disaster will be their first and only exposure. Moreover, as pointed out by Simon et al., traditional disaster exercises do little to provide experience or even the ability to assess the capacity of a hospital to provide care demanded by such a circumstance.

Furthermore, it is not possible to apply the experiences directly from one catastrophic event to the next. The principal limiting factor is the non-uniform use of the descriptors which express the nature and magnitude of the event or of how it was managed. As I proceeded through the previous volumes of Prehospital and Disaster Medicine, I became increasingly aware of the repeated use of identical descriptors to describe distinctly different elements of the responses: "disaster" is used interchangeably with multiple casualty incidents, mass casualties, and catastrophe; "phases" and "stages" are interchanged without definitions of either term; etc. Without common, universally accepted definitions, it is not possible to categorize and analyze one event relative to any other events. If this quandary persists, the field will remain a "body of notions" rather than a scientific discipline comprised of clearly defined basic concepts.

The tools for developing such expertise through comprehensive studies are at hand. These techniques are described in the international and multidisciplinary methods utilized for the research described in the preliminary report by Klain et al. Together with the methods described by Glittenberg in the last issue, both qualitative and quantifiable research can be acquired which promote definitions of patterns of injury and applicable resuscitology and can identify common elements in similar or even distinctly different events. But in order to allow the findings to be transferable from one awful experience to another, they must be described in a common, easily understood language. Therefore, I would like to propose a set of basic descriptors.

Manni and Magalini define the medical disaster as "a lack of proportion between the number of persons who must receive medical care and the available means to do so". In other words, a disaster has occurred if some part or all of the medical care capabilities are overwhelmed no matter to what degree or how temporary.There are three basic terms which are essential to describe a given disaster:

1. TYPE: The descriptors which define the nature of a disaster through description of the causative factor (s), the patterns of damage sustained (especially human), and the character of the environment in which the event has occurred indicate the type of disaster encountered. An earthquake with multiple structural failures and persons buried in the rubble generates types of responses which will be different than would be the responses that would result following a leak of toxic chemicals. 
Additional work is needed to categorize the types of disasters in accordance with the needed responses.

2. SCOPE: The scope of a disaster must describe the actual or expected number of persons injured and/or missing as well as the area involved, the nature of the terrain, and the possible means of access to the area in which the event has occurred. The magnitude of the response, including the quantity of resources which must be mobilized, is dependent upon the scope. The scope can vary from a multiple casualty incident which can be managed locally, to a train wreck as described by Wiles et al., in which the resources of a broader area than available in the local EMS system had to be mobilized, to the Armenian earthquake which required mobilization national and international resources. The scope must be defined as early as possible since the magnitude of the response demanded is dependent on the scope. The combination of the type and the scope of a disaster determine the nature of the responses which are mounted.

3. STAGES AND PHASES: The third major classifier indicates which and where specific resources are required. For example, there is little need for a plastic surgeon in the impact zone following an earthquake. Most agree that there are numerous stages associated with the responses to a disaster. Unfortunately, there is little agreement as to how such stages should be defined. In addition, there may be many subclassifications or phases of each stage. For example, prehospital emergency medical care is a stage of care delivered. It consists of at least the following phases: 1) Search and rescue; 2) Life-Saving First Aid; 3) Extrication; 4) Advanced Life Support; 5) Transportation to a receiving medical facility, etc. Additional phases may exist within the prehospital stage and must be defined as clearly as is possible. Other stages may include the events which occur at: the receiving facilities; preparation for and during interhospital transfers; the provision of tertiary care and prolonged life support; and others. Perhaps the most important of these stages is prevention.

An example of the importance of clearly defined stages as well as phases within a given stage is that it makes little sense to continue to convey specialist physicians to the scene-particularly those who are not accustomed to operating in the field setting (Pepe et al.). The physicians who initially arrived at the disaster site in Leninkanin quickly realized they were out of their element and wisely returned to the hospital. Specific resources are demanded by specific stages and phases within the specific stage. This allows the correct resources to be directed at the most appropriate phase within each stage.

A stage may be quite specific and yet overlap in time with other stages. Phases within a stage also may overlap. The duration of each will depend upon the scope and type of the disaster. However, it seems clear that regardless of the type and scope of the disaster, certain stages will be similar. Therefore, it is important to study carefully each disaster in the terms suggested and to identify stages and phases which are similar so that techniques learned in one setting maybe transferred appropriately to disasters of different types and scopes.

Without a standard nomenclature, we will continue to be restrained to anecdotal descriptions of these unfortunate and very stressful situations, the ability to grow from each experience will be limited, and Disaster Medicine will remain a series of "notions" and will not develop as a science. The responsibilities associated with the delivery of medical care for the victims of a disaster are awesome. We are on the brink of a revolution in disaster management. It may be helpful to meld the factions of the world into at least one common pathway: disasters pose a threat that faces each of us regardless of our system of beliefs or government. But in order to do so, we first must speak the same language. This is but a beginning of a new language and science. 